\title{
Epidemiological Approach for Data Survivability in Unattended Wireless Sensor Networks
}

\author{
Jacques M. Bahi, Christophe Guyeux, Mourad Hakem, and Abdallah Makhoul
}

November 20, 2018

\begin{abstract}
Unattended Wireless Sensor Networks (UWSNs) are Wireless Sensor Networks characterized by sporadic sink presence and operation in hostile settings. The absence of the sink for period of time, prevents sensor nodes to offload data in real time and offer greatly increased opportunities for attacks resulting in erasure, modification, or disclosure of sensor-collected data. In this paper, we focus on UWSNs where sensor nodes collect and store data locally and try to upload all the information once the sink becomes available. One of the most relevant issues pertaining UWSNs is to guarantee a certain level of information survivability in an unreliable network and even in presence of a powerful attackers. In this paper, we first introduce an epidemic-domain inspired approach to model the information survivability in UWSN. Next, we derive a fully distributed algorithm that supports these models and give the correctness proofs.
\end{abstract}

\section{Introduction}

Unattended Wireless Sensor Networks (UWSNs), which have been introduced by Di Pietro et al. in [8], are WSNs characterized by the sporadic presence of the sink. These UWSNs are useful for instance to detect poaching in a national park, or as a monitoring system to check the pressure of an underground pipeline, as stated in [18. In such networks, nodes collect data from the area under consideration, and then they try to upload all the stored data when the sink comes around. It is motivated by the scenarios where only historical information or digest data, not real-time data, are of interest. For example, what is average temperature during last three months; what is the highest and lowest humidity degree during last 24 hours; or more specifically, what is average content of a chemical element in soil during last half year [26.

Due to the absence of a direct and alive connection with the sink, these networks are more subject to malicious attacks than traditional WSNs. Sensor nodes may malfunction due to some threats, e.g., physical failure such as melting, corroding or getting smashed, and more sophisticated, mobile adversary attacks. Some data may be lost, erased or modified before the arrival 
of the mobile collectors, which significantly affects functionalities of UWSNs. Therefore, the critical issue for UWSNs is how to maximize information survivability [16]: the dimension of the area is often prohibitive in such networks, while the absence of the sink facilitates the work of attackers. Data or information survivability consists on preserving data for a long period of time in the face of attacks, which is crucial for designing safe UWSNs.

To ensure data survivability, cryptographic or noncryptographic approaches can be used. End-to-end encryption schemes that support operations over cypher-text have been proved important for private party sensor network implementations or other security schems [1, 23, 24. Unfortunately, nowadays these methods are very complex and not completely suitable for sensor nodes having limited resources. Non-cryptographic approaches are suitable for lowcost sensors, that do not have the capability to execute computational intensive calculation. In this paper, we will focus on non-cryptographic approaches for data survivability. We propose an epidemic-domain inspired approach to model the information survivability in UWSN.

The model we present here is based on both SIR (Susceptible - Infected Recovered) and SIS (Susceptible - Infected - Susceptible) models. A node is susceptible to a data item when it is online and functioning normally; he can receive the information that must survive. Intuitively, the model we focus on resembles an SIR model studied previously in [10, 18. Our novelty is that we study arbitrary dynamic network topologies instead of static networks. In a next step, we provide a fully distributed algorithm which supports/covers different epidemic models. The aim of this algorithm is to ensure data survivability in UWSN by maintaining a subset of safe nodes in working state while replacing/locking the attacked ones when needed.

The remainder of the paper is organized as follows: Section 2 briefly reviews the related work. The SIR model for Data Survivability in UWSNs is presented in Section 3 Section 4 details the proposed epidemic schemes in a comparatively manner. We present in section 5 the design and analysis of the proposed epidemic algorithm and give the proofs. The next section is devoted to numerical simulations. Finally, Section 7 concludes this research work.

\section{Previous work}

Wireless sensor networks have attracted a lot of interest over the last decade, for quick and efficient aggregation of information [4, 3], for understanding "trust" and "distrust" in online networks [2, 1, 11, and in several other areas. In most previous researches on WSNs, sensed data are assumed to be collected in a realtime manner and sensor nodes always connected to the sink that is generally the only unconditionally trusted entity. In order to extending the network lifetime a possible solution presented in the literature and consists on using mobile sinks or robots to perform several tasks instead of the nodes themselves. For instance, the authors in [6, 5] propose a mobile beacon based approach to localize sensor nodes and ensure the network coverage. In [25], a distributed algorithm is pro- 
posed in order to reduce the overhead message by using only local information and assisted by mobile sinks. Recently, a number of approaches exploiting sink mobility for data collection in WSNs have been proposed [14, 22, 21, 15]. The main objective of these work is to reduce the energy consumption by optimizing the number of communications between nodes. In these approaches only single hop communication is required between nodes and the mobile sinks. Otherwise, in some cases and scenarios sensor nodes can not be connected for a short or long period of time and the network is left unattended while sensor nodes cannot offload data in real time. Therefore, sensor nodes wait for the new passing of a mobile sink for examle to send their data. Unattended Wireless Sensor Networks (UWSNs), have been introduced by Di Pietro et al. in 8, where adversaries can compromise some sensor nodes and selectively destroy data. Other works have studied this problem in order to prevent such attacks, and ensure the "data survivability" in UWSN.

The authors in [17 proposed a method to face an adversary that indiscriminately erases all sensor data, and then in [9] cryptographic techniques that prevent the adversary from recognizing data that it aims to erase have been introduced. Sensor cooperation to achieve self-healing in stationary UWSNs has been explored in [16. 20] proposes a new strategy based on the concept of secure multi-party protocols. The main advantages of the proposed strategy are not limited to the security, since it preserves privacy, enables the use of data aggregation and enforces a level of trust among nodes, which collaborate to compute aggregation functions. In [19], the authors propose a dependable and efficient data survival scheme to maximize the data survival degree upon data retrieval. This technique makes use of computational secret sharing to achieve fault tolerance and compromise resilience, and uses network coding to further improve communication efficiency. In [7, the authors focus on the conditions under which a sensor node can survive in an unreliable network. They propose and solve the problem using non-linear dynamical systems and fixed point stability theorems.

The epidemiology community has developed the so-called SIR and SIS models [10, 18] of infection. The SIS model (Susceptible - Infected - Susceptible) is suitable for, e.g., the common flu, where nodes may be infected, healed (and susceptible), and infected again. The SIR model (Susceptible - Infected - Recovered) is suitable for, say, mumps, where a node, after being infected, becomes recovered (with life-time immunity). SIS, SIR, and SIRS models have been investigated by authors of these research works, in order to derive the parameters that can assure information to survive. In these articles, $S(t)$ compartment is constituted by sensors that do not possess the datum at time $t$, while $I(t)$ is the compartment of sensors that possess it. Finally, the $R(t)$ compartment is constituted by sensors that have been compromised by the attacker.

On the other side and surprisingly, the authors in [10, 18] never consider that in a wireless sensor network, nodes' energy is provided by a battery that can be emptied due to data acquisition, transmission, or simply functioning cost of keeping alive. More precisely, the topology of the networks they consider is static, the network's lifetime is unbounded, and sensors cannot die due to 
empty batteries. Indeed, their work is more related to unattended wired sensor networks, on main power but not with a battery as $S+I$ (SIS model) or $S+I+R$ (SIR and SIRS models) are constant. Our intention is to deepen their interesting work, by bringing their proposal from wired sensor networks to WSNs, refining theirs models, and producing more theoretical results on each model.

\section{A SIR model for Data Survivability in UWSNs}

\subsection{Introducing the Kermack \& McKendrick model}

In this section, the SIR model formerly presented in [10, 18, is firstly recalled. Then, consumption hypotheses underlined in this model are precised while theoretical results on the behavior of the compartments of the network are further investigated.

In unattended wireless sensor networks the presence of the sink is sporadic. However the duration between two visits of the sink to the network (its absence) can sometimes be considered negligible, in a first approximation, compared to the time required to empty a sensor battery. In such UWSNs, the death processes of sensors can be neglected if the aim is to study the immediate consequences of an attack between two visits of the sink. Under such an assumption, the global network can be divided in three compartments, namely the sensors $S$ susceptible to receive the datum of interest (intrusion detection, etc.), the ones that currently store it $I$, and the recovered sensors $R$ that have been compromised by the attacker: their stored datum has been recovered.

Suppose now that between $S$ and $I$, the transmission rate is $b I$, where $b$ is the contact rate, which is the probability of transferring the information in a contact between a susceptible sensor and a sensor having the datum. Indeed, as proven by Di Pietro et al., such a situation occurs when then wireless sensor network is composed by $n$ sensor, and if each sensor forwards the datum with probability $\frac{\alpha}{n}$ [10, 18] ( $\alpha$ is the transition rate).

Suppose additionally that the rate to pass between $I$ and $R$, is $c$ : the attacker is able to individuate the sensors containing the target information, and to destroy each of them with this probability $c$. Notice that, if the duration of the information survivability is $D$, then $c=\frac{1}{D}$, as a sensor experiences one recovery in $D$ units of time.

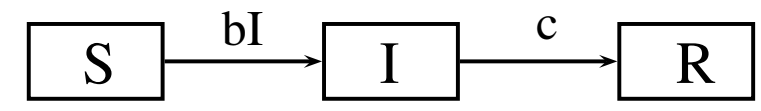

Figure 1: SIR model

Under such hypotheses and as stated in [10, 18, the sensors population follows the so-called SIR model of Kermack \& McKendrick [13] depicted in Figure 1. Remark that the total sensors population is equal to $N=S+I+R=$ $S_{0}+I_{0}+R_{0}$, which is a constant: the number of awaken, alive sensors does not 
evolve. In particular, only two of the three populations of sensors have to be studied.

\subsection{Firsts theoretical results}

Consider now that $x(t)=\frac{X(t)}{N}$ denotes the fraction of individuals in the compartment $X$. The SIR model can be expressed by the following set of ordinary non-linear differential equations:

$$
\left\{\begin{array}{l}
\frac{d s}{d t}=-b i s \\
\frac{d i}{d t}=b i s-c i \\
\frac{d r}{d t}=c i
\end{array}\right.
$$

Obviously, the typical time between transmissions is $T_{t}=b^{-1}$ while the typical time until attack when having the information is equal to $T_{e}=c^{-1}$. Thus

$$
\frac{T_{t}}{T_{e}}=\frac{c}{b}
$$

is the average number of transmissions between a sensor having the datum and others before it lost this information due to the attacker. Such a statement explain why, in the SIR historical model, the dynamics of the infectious class depends on the reproduction ratio defined by

$$
R_{0}=\frac{b}{c}
$$

which corresponds here to the expected number of new informed sensors (socalled "secondary infections") providing a single sensor with the datum where all sensors are susceptible. Furthermore, direct standard analysis manipulations (variables separation and then integration) lead to the following form for the susceptible sensors compartment: $s(t)=s(0) \exp \left(-R_{0}(r(t)-r(0))\right)$.

As $\frac{d i}{d t}=\left(R_{0} s-1\right) c i$, if the basic reproduction number satisfies $R_{0}>\frac{1}{s(0)}$, there will be an information outbreak with an increasing number of sensors with the datum. In other words, $R_{0}$ determines whether or not the information will spread through the network.

All these facts are summarized in the proposition below.

Proposition 1 Consider a sensor network that aims to monitor a given area, and that has to spread an alert or an information to a sink, whose presence is sporadic. Suppose that an attacker tries to remove the datum in sensors' memory, and that:

1. all sensor activities are negligible, in terms of energy, 
2. when a sensor has the datum, it spreads the information to its neighbors with a probability $b$, until being attacked.

Denote by $T_{t}$ the typical time between transmissions, $T_{e}$ the typical time an informed sensor loses its information due to the attacker, and by $s(0)$ the initial fraction of susceptible sensors. So the information will spread through the network if and only if $T_{t}<s(0) T_{e}$.

In other words, this proposition states that if the reproduction ratio is greater than one, then an "epidemic" occurs since the prevalence (the infected ratio) increases to a peak and then decreases to zero. Otherwise there is no epidemic since the prevalence decreases to zero.

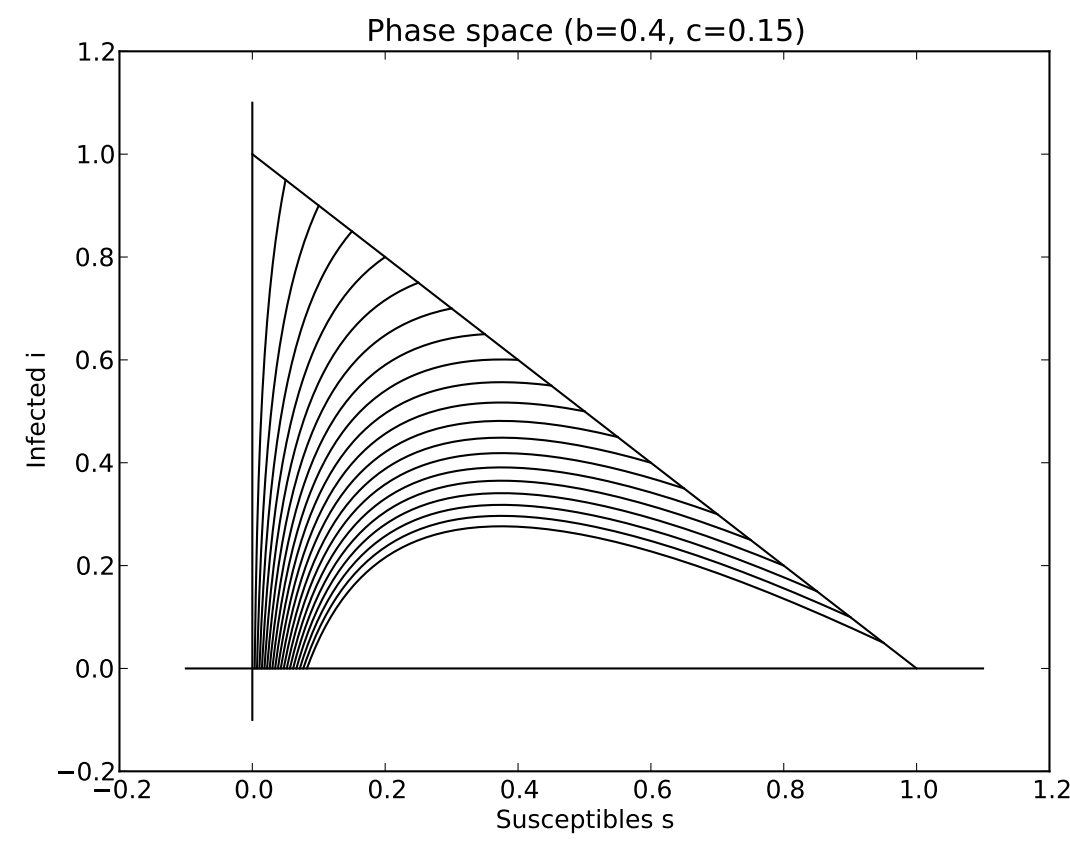

Figure 2: Phase space $(s, i)$ with $b=0.4, c=0.15$ (SIR model).

It is possible to be more precise in the formulation of Proposition 1 following an approach similar to [12].

Proposition 2 The fraction $s(t)$ of sensors susceptible to receive the information is a decreasing function. The limiting value $s(\infty)$ is the unique root in $\left(0, \frac{T_{e}}{T_{t}}\right)$ of the equation

$$
1-r(0)-s(\infty)+\frac{T_{e}}{T_{t}} \ln \left(\frac{s(\infty)}{s(0)}\right) .
$$


Additionally,

- if $T_{t} \geqslant s(0) T_{e}$, then the fractional number $i(t)$ of sensors having the datum decreases to zero as $t \rightarrow \infty$,

- else $i(t)$ first increases up to a maximum value equal to $1-r(0)-\frac{T_{e}}{T_{t}}\left(1+\ln \left(\frac{s(0) T_{t}}{T_{e}}\right)\right)$ and then decreases to zero as $t \rightarrow \infty$, where ln stands for the natural logarithm.

Proof The triangle $T=\{(s, i) \mid s \geqslant 0, i \geqslant 0, s+i \leqslant 1\}$ is positively invariant, since from the SIR equations, it holds: $s=0 \Rightarrow s^{\prime}=0, i=0 \Rightarrow i^{\prime}=0$, and $s+i=1 \Rightarrow(s+i)^{\prime}=-c i \leqslant 0$. Furthermore, points on the $s$ axis where $i=0$ are equilibrium ones, unstable for $s>1 / R_{0}$ and stable otherwise. $s$ is decreasing and positive due to this invariance and because $\frac{d s}{d t}=-b i s$, so an unique limit $s(\infty)$ exists. Similarly, $r^{\prime}(t)=c i \geqslant 0$ and $r \leqslant 1$ then $r(\infty)$ exists. As $s+i+r=1, i(\infty)$ exists too. To prove that this limit is null, we only remark that if $i(\infty)>0$, then $r(\infty)=\infty$ (because $r^{\prime}>\frac{c i(\infty)}{2}$ for sufficiently large $t$ ), which is impossible, as $r \leqslant 1$. Finally, the equations of the proposition are derived from $\frac{d s}{d i}=\frac{c}{b s}-1$.

The phase space of the solutions of the SIR system with given parameters is provided in Figure 6 while the evolution of $s$ and $i$ is depicted in Figure 10. The results presented in this section hold for a transition rate between susceptible and informed sensors having the form $F=a i$, which thus represents the force of information. Nonlinear forces of information, or infection, can be investigated too, to model more realistically the information survivability.

\subsection{Another understandings for the Recovered compart- ment}

In the previous section, the $R$ compartment was constituted by sensors that have been compromised by the attacker, which will be referred in what follows as situation 1. It is possible to attribute at least two other understandings to this compartment, for an unattended wireless sensor network whose lifetime is dependent on energy consumption and in absence of attacks.

This compartment can be constituted by dead sensors, when considering that the sole action on the energy is the information transmission, and that the unique way to death for a sensor is to have too much transmitted the datum. In other words, in this Situation 2, sensors send information messages to their neighbors until emptying totally their batteries. The sink will receive the information when it will interrogate the network at time $t$ if $I(t) \neq 0$.

A third situation can be considered without any changes in formalization, except redefining the meaning of the $R$ compartment. Indeed, it can be interesting to consider that a sensor is first susceptible to receive an information message 


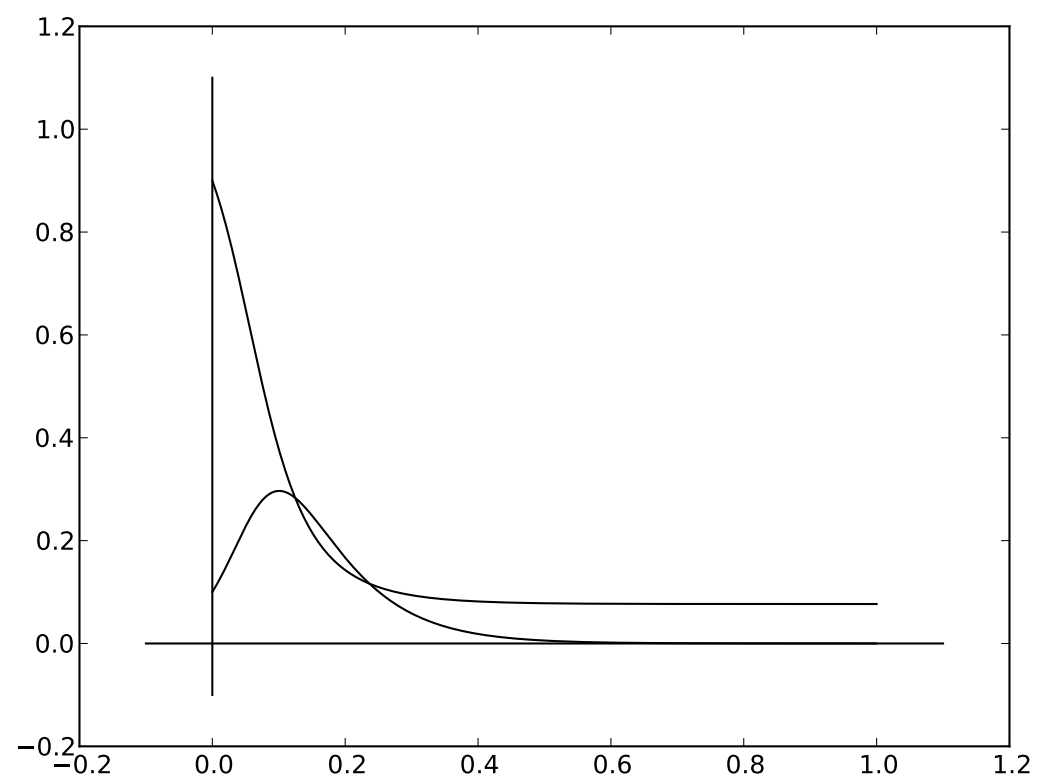

Figure 3: Evolution of the fractions $s$ and $i$ of susceptible and having the datum sensors with $b=0.4, c=0.15, s(0)=0.9$, and $i(0)=0.1$ (SIR model).

for a while, then in a second time it has and transmit the information, before finally entering into the third age of its life, the recovered state in which it will lose its ability to transmit the information. Materials of the previous section tackles too this scenario, when considering the network lifetime sufficiently large compared to information spreading, in order to neglect sensors' death due to energy consumption. The question here is to determine the quantity of informed sensors on large timescales.

Let us now explain how to extend such a compartmental study for data survivability in wireless sensor networks to well-known SIS models.

\subsection{A few words about SIS models}

Other compartmental divisions of the set of sensors can be investigating, leading for instance to a SIS epidemic model [13]. This latter assumes only two compartments named Susceptible (S) and Infected (I). Transitions between these compartments are represented in Figure 4. An individual that is susceptible to a disease becomes infected with a certain probability $a$, while an infected individual immediately becomes susceptible once (and if) it is cured of an infection (which happens with probability $b$ ). Note that a healthy individual can 
contract a disease only if it is in contact with a sick one. Thus, the evolution of this system is completely described by the following two differential equations (total sensor population: $P=S+I=S_{0}+I_{0}$, which is a constant).

$$
\begin{cases}\frac{d I}{d t}=a S I-b I & I(0)=I_{0} \\ \frac{d S}{d t}=b I-a S I & S(0)=S_{0}\end{cases}
$$

The SIS model may be treated the same as the SIR model, which has been detailed in this section. For the sake of concision, and as this study does not raise any complication, this model will be left as an exercise, while energy consumption will now be investigated in the next section.

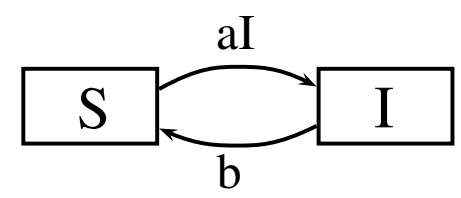

Figure 4: SIS model

\section{Considering Energy Consumption for Data Sur- vivability in UWSNs}

In a large amount of situations, energy consumption and the death of sensors cannot be neglected, this is why a "natural" death rate for all compartments is introduced in this section. Such an approach generalizes the models presented previously.

\subsection{A SIR model with natural death rate}

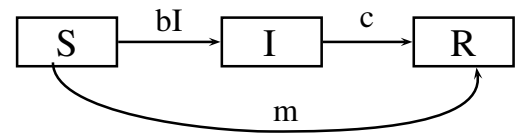

(a) Situation 2

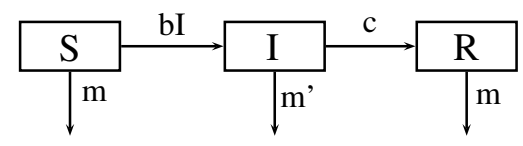

(b) Situations 1 and 3

Figure 5: SIR models with natural death rate

The previous section considers that all sensor activities are negligible, in terms of energy, except the transmission of information in situations 2 and 3 , which is reasonable in a first approximation. It is however possible to refine the SIR model in these two last situations, in order to consider that sensors' energy decreases too in absence of information transmission. 


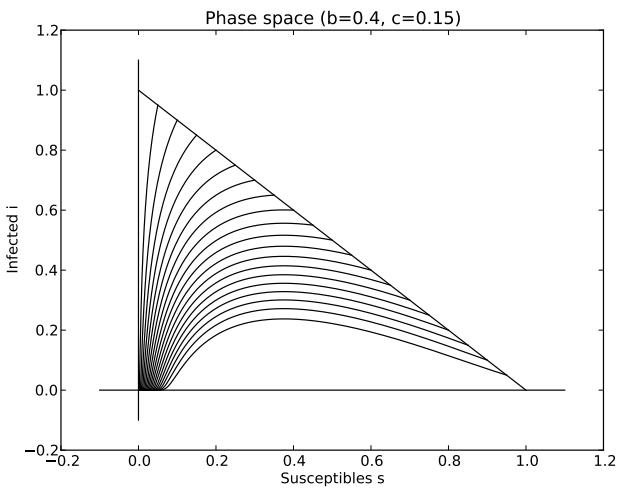

(a) Situation 2

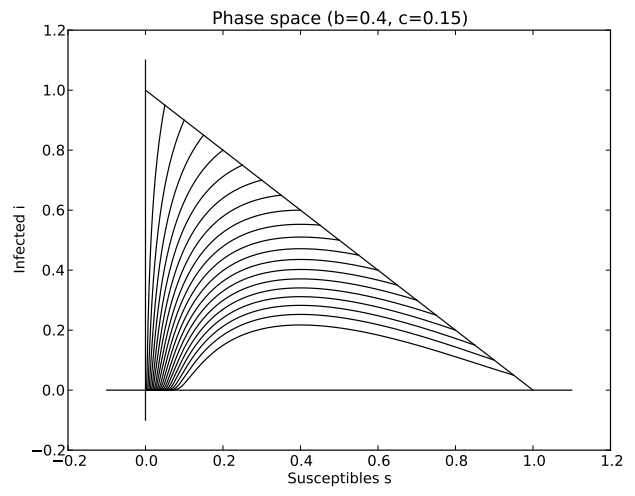

(b) Situations 1 and 3

Figure 6: Phase space $(s, i)$ with $b=0.4, c=0.15, m=0.01$, SIR model with natural death rate in Situation 3.

In Situation 2, the $R$ compartment of the SIR model is constituted by dead sensors. This compartment is populated by susceptible nodes that have naturally died (death rate $m$ ) without having received the datum and by sensors of the $I$ compartment which die at another rate $c$ supposed to be greater than $m$, as they have to transfer the datum, an energy-consuming task. This situation is depicted in Figure $5(\mathrm{a})$

In the two other situations investigated in this research work, the $R$ compartment is constituted by living sensors that do not transmit the datum anymore, either because they have been corrupted and thus have lost it (first situation), or because their batteries is preserved (third one). This new situation is closed to the SIR model of Figure 1, except that a the new network is characterized by a death rate for each sensors compartment (see Figure $5(\mathrm{~b})]$. Notice that the death rate $m^{\prime}$ of the $I$ compartment is a priori different from the one of $S$ and $R$ compartments, as it is reasonable to suppose that the datum transmission implies more energy consumption. However, setting $m^{\prime}=m$ is possible too.

The SIR model of Equation (1) can be adapted as follows for Situation 2:

$$
\left\{\begin{array}{l}
\frac{d s}{d t}=-b i s-m s \\
\frac{d i}{d t}=b i s-c i \\
\frac{d r}{d t}=c i+m s
\end{array}\right.
$$


while it has the following form in Situations 1 and 3:

$$
\left\{\begin{array}{l}
\frac{d s}{d t}=-b i s-m s \\
\frac{d i}{d t}=b i s-c i-m^{\prime} i \\
\frac{d r}{d t}=c i-m r .
\end{array}\right.
$$

Let us now investigate the long-term behavior of these models. Regarding Situation 2, it is natural to think that, for large timescales, all sensors will take place in the third $R$ compartment of died sensors, as all the batteries are continually emptied (either due to natural consumption or because of the information transmission). This can be easily proven by considering that in an equilibrium point $\left(s^{*}, i^{*}, r^{*}=1-s^{*}-i^{*}\right)$, we have $\frac{d s}{d t}=\frac{d i}{d t}=\frac{d r}{d t}=0$, and so

$$
\left\{\begin{array}{l}
\left(b i^{*}+m\right) s^{*}=0 \\
\left(b s^{*}-c\right) i^{*}=0 \\
c i^{*}+m s^{*}=0
\end{array}\right.
$$

As $c>0, m>0, i^{*} \geqslant 0$, and $s^{*} \geqslant 0$, we can conclude from the third equation above that $s^{*}=i^{*}=0$, and so $r^{*}=1$. The Jacobian is equal to

$$
J(s, i, r)=\left(\begin{array}{ccc}
-b i-m & -b s & 0 \\
0 & b s-c & 0 \\
m & c & 0
\end{array}\right)
$$

and its characteristic polynomial in $(0,0,1)$ is $\lambda(\lambda+c)(\lambda+m)$. The eigenvalues being negative, the equilibrium $(0,0,1)$ is attractive. These results are summarized in the following proposition.

Proposition 3 Consider an unattended wireless sensor network divided in three sets of sensors, the first category $S$ being susceptible to receive a given datum, the second one $I$ having and transmitting this latter, and the third one $R$ being constituted by dead sensors.

Suppose that the death rate is $m$ for $S$ compartment and $c$ for I's one, and that the transmission rate is bI between $S$ and I. In that situation, for all initial condition and for all positive parameters $b, c$, and $m$, the system is convergent to the equilibrium point $(0,0,1)$.

In particular, in that situation, the datum cannot survive a long time in the UWSN.

Equation 3 can be resolved similarly: from $b i^{*} s^{*}+m s^{*}$, we deduce that $s^{*}=0\left(\right.$ as $\mathrm{b}>0, \mathrm{~m}>0$, and $\left.i^{*} \geqslant 0\right)$. So $b i^{*} s^{*}-c i^{*}-m^{\prime} i^{*}$ implies that $i^{*}=0$ too. Finally, from the third line, we conclude that $r^{*}=0$. Eigenvalues of the characteristic polynomial of the Jacobian in $(0,0,0)$ are $-m$ and $-c-m^{\prime}$, 


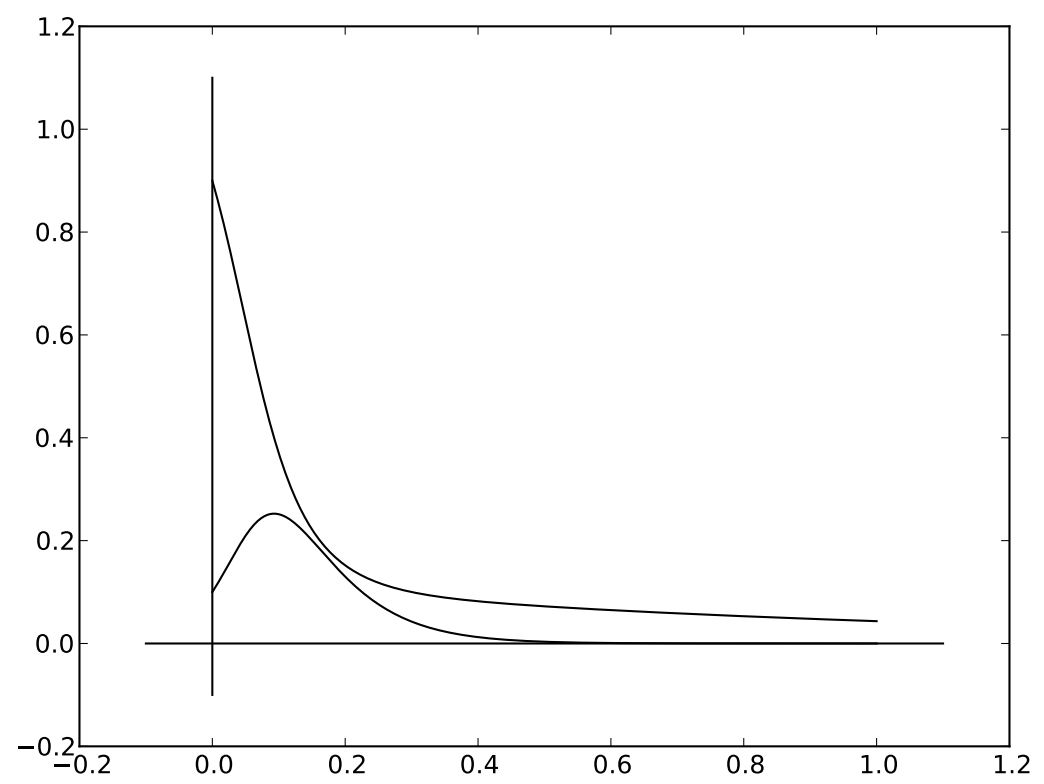

Figure 7: Evolution of the fractions $s$ and $i$ of susceptible and having the datum sensors with $b=0.4, c=0.15, m=0.01, s(0)=0.9$, and $i(0)=0.1$, SIR model with natural death rate in Situations 1 and 3.

which are negative. So this equilibrium point is attractive too, and a similar proposition than previously can be formulated, with the same conclusion, both for Situations 1 and 3. Phase spaces for the three situations are provided in Figure 6 while Fig. 10 depicts the evolution of the fractions $s$ and $i$ in Situations 1 and 3 .

To put it in a nutshell, to achieve data survivability in UWSNs, the birth of awaken sensors must be considered, which is the subject of the next subsection.

\subsection{A scheduling process in data survivability}

\subsubsection{A first natural approach}

A first idea to realize a more realistic model of an unattended wireless sensor network is to establish a scheduling process of the sensor nodes, in order to enhance data survivability for a long period of time. Considering the SIR model, such a process leads to the division of each compartment in two parts, corresponding respectively to awaken and to sleeping sensors, as depicted in Figure 8 


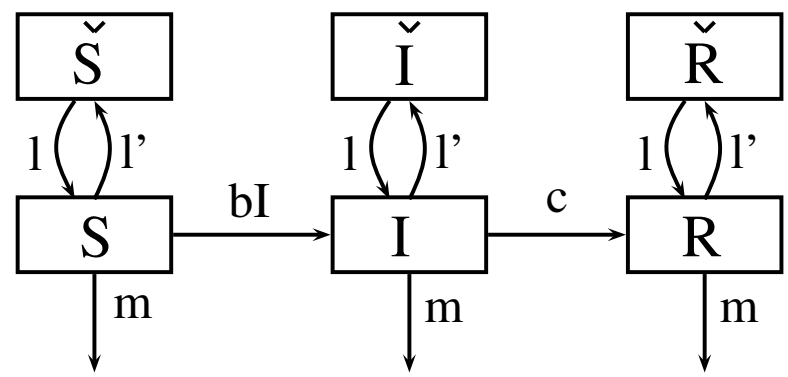

Figure 8: SIR model with natural death rate and sleeping nodes

Such a model can be reformulated as follows:

$$
\begin{cases}\frac{d s}{d t}=l \check{s}-l^{\prime} s-b i s-m s & \frac{d \check{s}}{d t}=-l \check{s}+l^{\prime} s \\ \frac{d i}{d t}=l \check{i}-l^{\prime} i+b i s-c i-m i & \frac{d \check{i}}{d t}=-l \check{i}+l^{\prime} i \\ \frac{d r}{d t}=l \check{r}-l^{\prime} r+c i-m r & \frac{d \check{r}}{d t}=-l \check{r}+l^{\prime} r .\end{cases}
$$

The equilibrium point $\left(s^{*}, \check{s}^{*}, i^{*}, \check{i}^{*}, r^{*}, \check{r}^{*}\right)$ is searched once again, it satisfies:

$$
\begin{cases}l \check{s}^{*}-l^{\prime} s^{*}-b i^{*} s^{*}-m s^{*}=0 & -l \check{s}^{*}+l^{\prime} s^{*}=0 \\ l \check{i}^{*}-l^{\prime} i^{*}+b i^{*} s^{*}-c i^{*}-m i^{*}=0 & -l \check{\check{r}}^{*}+l^{\prime} i^{*}=0 \\ l \check{r}^{*}-l^{\prime} r^{*}+c i^{*}-m r^{*}=0 & -l \check{r}^{*}+l^{\prime} r^{*}=0 .\end{cases}
$$

Obviously, $l \check{s}^{*}=l^{\prime} s^{*}, l \check{i}^{*}=l^{\prime} i^{*}$, and $l \check{r}^{*}=l^{\prime} r^{*}$, and so:

$$
\left\{\begin{array}{l}
\left(b i^{*}+m\right) s^{*}=0 \\
\left(b s^{*}-c-m\right) i^{*}=0 \\
c i^{*}-m r^{*}=0
\end{array}\right.
$$

If $s^{*} \neq 0$, then $b i^{*}+m=0$, which is impossible if it is reasonably supposed that each rate is $>0$. So $s^{*}=0$, which implies that $i^{*}=0$, and so $r^{*}=0=\check{r}^{*}=$ $\check{i}^{*}=\check{s}^{*}$.

To sum up, in the unique stable equilibrium point, the number of informed sensors is null, and we face a data loss. This problem is solved in the next section, by considering that nodes never go to sleep.

\subsubsection{Achieving data survivability using birth and death rates}

Consider now a new approach proposed to solve the loss of information in the former scheduling process. In this second approach for scheduling, sensors can 


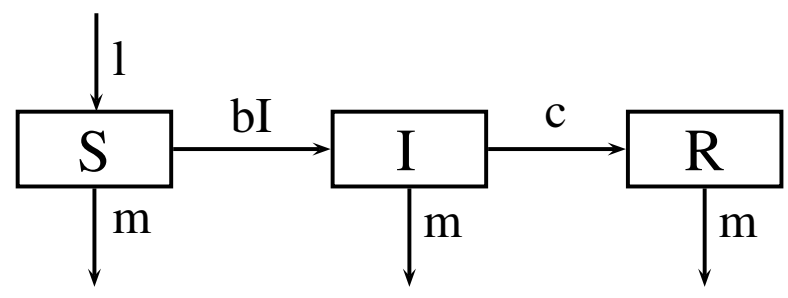

Figure 9: SIR model with natural birth and death rates

only be awaken (we never order them to sleep). It is supposed that a sufficiently large number of sensors are available, and the question is to determine if it is possible to determine the lowest birth rate to achieve data survivability for a long period of time, even in presence of an adversary.

To do so, it is supposed that, at the initial stage, only a small part of the sensors nodes is awakened. New sensors are then awakened periodically during the network's service at a rate $l$, repopulating by doing so the $S$ compartment (they never go to sleep). Along with this birth rate, a natural death rate $m$ is considered for each of the three kind of sensors, while the $R$ compartment is for corrupted sensors in the original situation 1, as depicted in Figure 9, Remark that such a model is compatible with living and awaken nodes that have stopped to transfer the information in Situation 3.

To model such a scenario requires to rewrite the first line of Equation (2), leading to the following system:

$$
\left\{\begin{array}{l}
\frac{d s}{d t}=l-b i s-m s \\
\frac{d i}{d t}=b i s-c i-m i \\
\frac{d r}{d t}=c i-m r
\end{array}\right.
$$

This updated system is the usual SIR model with vital dynamics, in which we have not supposed the birth and death rates equal. It is possible to show that the problem is well formulated, as the triangle $T=\{(s, i) \mid s \geqslant 0, i \geqslant 0, s+i \leqslant 1\}$ still remains positively invariant.

A study of this system supposes to consider the Poincaré-Bendixon theorem in phase space and the use of Lyapunov functions [12. It can however be understood by considering what will happen to the information in a long run: will it die out or will it establish itself in the network like an endemic situation in epidemiological models? The long-term behavior of the solutions, which depends largely on the equilibrium points that are time-independent solutions of the system, must be investigated to answer this question. Since these solutions do not depend on time, we have $s^{\prime}(t)=i^{\prime}(t)=r^{\prime}(t)=0$, which leads to the 
system:

$$
\left\{\begin{array}{l}
0=l-b i s-m s \\
0=b i s-(c+m) i \\
0=c i-m r
\end{array}\right.
$$

$r=\frac{c}{m} i$ from the last equation, and either $i=0$ or $s=\frac{c+m}{b}$ from the second one. On the one hand, if $i=0$, then $r=0$, and $s=\frac{l}{m}$ from the first equation. This leads to the equilibrium solution

$$
\left(\frac{l}{m}, 0,0\right)
$$

As the number of sensors having the datum is 0 in this point, it means that if a solution of the system approaches this equilibrium, the fraction $i$ will approach 0, and the datum tends to disappear from the network: an information-free equilibrium. Remark that the existence of this equilibrium is independent of the parameters of the system: it always exists.

On the other hand, if $i \neq 0$, then $s=\frac{c+m}{b} \neq 0$ from the second equation, and $\frac{l}{s}=b i+m$ according to the first equation. Substituting $s$ and solving for $i$, we find

$$
i=\frac{b l-m(c+m)}{b(c+m)}=\frac{R_{0} l-m}{b}
$$

with $R_{0}=\frac{b l}{m(c+m)}$, which is a positive number iff $R_{0}>1$.

$R_{0}$ is the reproduction number of the information, which tells us how many secondary informed sensors will one informed sensor produces in an entirely susceptible network, as: - a network which consists of only susceptible nodes in a long run has $\frac{l}{m}$
sensors;

- $c+m$ is the rate at which sensors leave the $I$ compartment. In other words, the average time spent as an informed sensor is $\frac{1}{c+m}$ time units.

- The number of data transmissions per unit of time is given by the incidence rate $b I S$. If there is only one informed sensor $(I=1)$ and every other sensor is susceptible $\left(S=\frac{l}{m}\right)$ then the number of transmissions by one "infected" node per unit of time is $\frac{b l}{m}$. 
So the number of data transmissions that one informed sensor can achieve during the entire time it is not attacked if all the reminded sensors are susceptible, is $\frac{b l}{m(c+m)}$, that is, $R_{0}$.

So if $R_{0}>1$, the number of sensors having the datum is strictly positive in this equilibrium solution: if some other solutions of the system approach this equilibrium as time goes large, the number of sensors having the datum will remain strictly positive, and the information remains in the network and becomes endemic.

These statements are summarized in the following proposition.

Proposition 4 If either $R_{0} \leqslant 1$ or $s(0)=0$, then any solution $(s(t), i(t))$ is convergent to the equilibrium without information $(1,0)$.

If $R_{0}>1$ then there are two equilibria: the non attractive informationfree equilibrium and the endemic equilibrium. This latter is attractive so that solutions of the ODE system approach it as time goes to infinity: the information remains endemic in the UWSN.

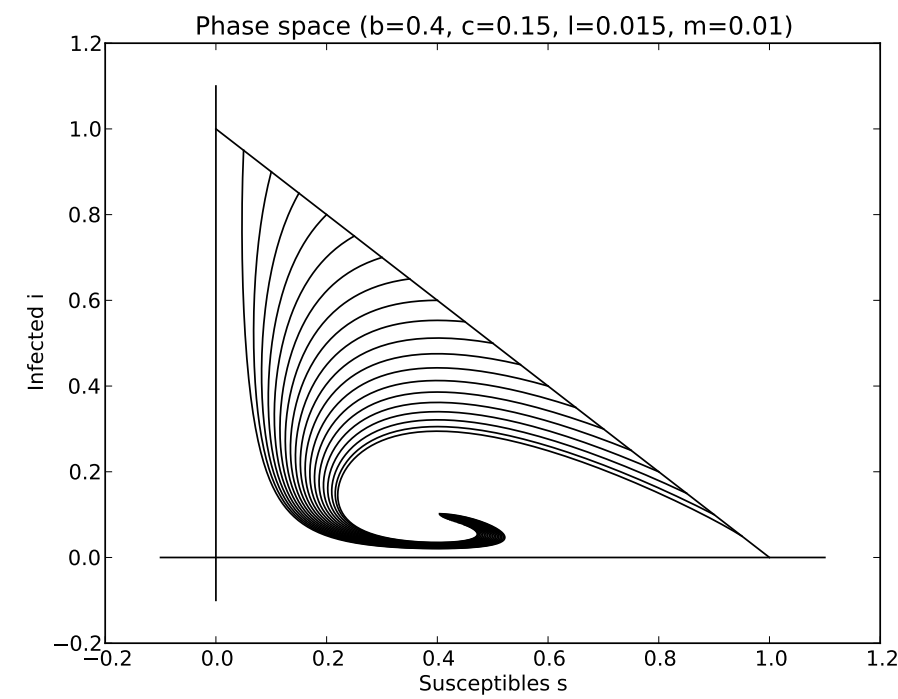

Figure 10: Evolution of the fractions $s$ and $i$ of susceptible and having the datum sensors, SIR model with natural birth and death rates $\left(R_{0}=3.75\right)$.

The attacker desire is to have $R_{0}<1$ to tend to an information-free equilibrium, whereas $R_{0}$ must be greater than 1 for the sink to face such attack. If the attacker has the opportunity to observe the network running a certain duration, then he or she can infer the values of parameters $b, c, m$, and $l$. Let $N$ be the number of data transmissions by one informed node per time unit, that 
is, $N=\frac{b l}{m_{1}}$. If the attacker is able to detect and infect the informed nodes in a time $\frac{m_{1}}{c+m}$ lower than $\frac{1}{N}$, then he or she is sure that $R_{0}<1$ : the data will not survive in the network. The sink interest, for its part, is to have $\frac{b l}{m}$ large and $\frac{1}{c+m}$ low, which can be achieved in the following manner:

- increasing the birth rate $b$,

- increasing the lifetime of sensors to reduce $m$,

- increasing the data transmission rate $b$, but $m$ increases when $b$ increases,

- if possible, reducing $c$ by considering countermeasures against data removal.

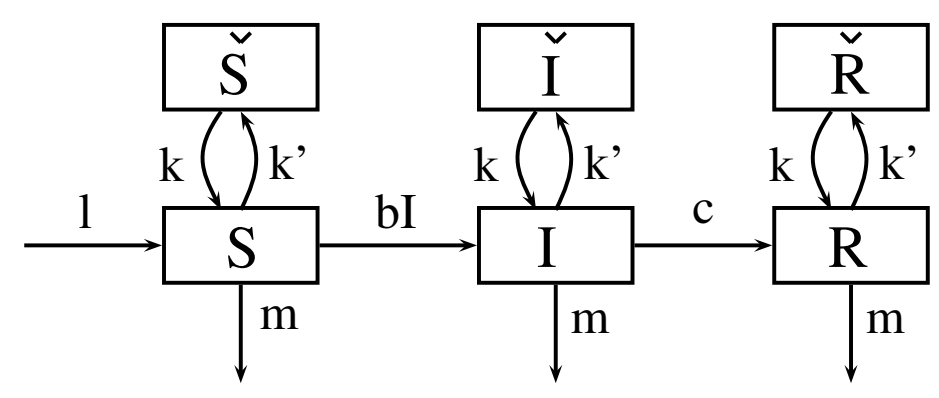

Figure 11: Global SIR model with natural birth and death rates, and sleeping nodes

Remark finally that this study is compatible with the situation depicted in Figure 11. in which awaken sensor nodes are allowed to go to sleep. Indeed this situation, which has not been detailed in this section to avoid making the text more cumbersome, introduces three new compartments $\check{S}, \check{I}$, and $\check{R}$ as in the previous section. However, as we focused on the future of the information in a long run, we only have to consider equilibrium points that are time-independent solutions of the system. As shown in the previous section, we obtain $\frac{d \check{s}}{d t}=$ $-k \check{s}+k^{\prime} s=0, \frac{d \check{i}}{d t}=-k \check{i}+k^{\prime} i=0$, and $\frac{d \check{r}}{d t}=-k \check{r}+k^{\prime} r$. Consequently, compartments $\check{S}, \check{I}$, and $\check{R}$ disappear in the final global system corresponding 
to figure 11

$$
\begin{cases}\frac{d s}{d t}=l+k \check{s}-k^{\prime} s-b i s-m s & \frac{d \check{s}}{d t}=-k \check{s}+k^{\prime} s \\ \frac{d i}{d t}=k \check{i}-k^{\prime} i+b i s-c i-m i & \frac{d \check{i}}{d t}=-k \check{i}+k^{\prime} i \\ \frac{d r}{d t}=+k \check{r}-k^{\prime} r+c i-m r & \frac{d \check{r}}{d t}=-k \check{r}+k^{\prime} r,\end{cases}
$$

and exactly the same Proposition 4 is obtained.

\section{The proposed algorithm}

In this section, a fully distributed algorithm which supports/covers different epidemic models is presented and theoretically analyzed. Our algorithm seeks to ensure data survivability by maintaining a necessary set of safe working nodes and replacing/locking attacked ones when needed.

In the following, we first focus on the legitimate state formulation and next, we present the algorithm which consists in only three rules and give the correctness proofs.

\subsection{Problem formalization}

Let $G=(V ; E)$ the graph modeling the sensor network, with $|V|=n$ and $|E|=m$. We assume sensor node identifiers to be unique. Recall that sensor node identifier is unique if and only if $i . I d \neq j$.Id holds for each $i, j \in V(i \neq j)$. A sensor node can be in one of these four states: working, probing, sleeping or locked.

We say that a sensor node $i$ is independent if

$$
\text { i.state }=\text { working } \wedge\left(\forall j \in N_{i}\right)(j . s t a t e=\text { sleeping } \vee \text { probing } \vee \text { locked })
$$

and that $i$ is dominated if

$$
(\text { i.state }=\text { sleeping } \vee \text { probing } \vee \text { locked }) \wedge\left(\exists j \in N_{i}\right)(j . s t a t e=\text { working })
$$

The legitimate state (let denote it $\mathcal{L}$ ) of the network is then expressed as follows:

$$
\forall i \in V: \text { i.state }=\text { working } \Rightarrow \text { i.compartment }=S \vee I
$$

In other words, each working node is either in $S$ or $I$.

The following notations are also given for the predicates of node $i$

- $A(i)$ : attacked neighbor: $\exists j \in N_{i}, j$.compartment $=R$

- $W(i)$ : working neighbor: $\exists j \in N_{i}$,j.state $=$ working

- $W^{*}(i)$ : working neighbor with lower Id: $\exists j \in N_{i}$,j.state $=$ working $\wedge i . I d>j . I d$

- $P^{*}(i)$ : probing neighbor with lower Id: $\exists j \in N_{i}, j$. state $=$ probing $\wedge i . I d>j . I d$ 


\subsection{The algorithm}

The proposed algorithm uses the following three rules:

$r_{1}$ :

if $i . s t a t e=$ probing $\wedge W(i)$ then

if $j$.compartment $=I$ then

i.compartment $\leftarrow I$ (*the datum is transferred/replicated to/on $i *$ )

end if

i.state $\leftarrow$ sleeping

end if

$r_{2}$ :

if $i . s t a t e=$ probing $\wedge\left(\neg W(i) \wedge \neg P^{*}(i) \vee A(i)\right)$ then

if $A(i)$ then

$j . s t a t e \leftarrow$ locked (*node $j$ remains locked until its healing/recovery*)

end if

i.state $\leftarrow$ working

end if

$r_{3}:$

if $i . s t a t e=$ working $\wedge W^{*}(i)$ then

if i.compartment $=S \wedge j$.compartment $=I$ then

i.compartment $\leftarrow I$ (*the datum is transferred/replicated to/on $i *$ )

end if

i.state $\leftarrow$ sleeping

end if

\subsection{Correctness proofs}

Lemma 1 If a node changes to the working state by $r_{2}$, then it remains in its state and will never execute a rule again until an eventual attack.

Proof Let $i$ be a sensor node that executes $r_{2}$. According to the preconditions of all rules, node $i$ can execute only rule $r_{3}$ in the next round. However, in order to do so, one of its neighbors would have to change into working state by $r_{2}$. This is impossible as long as node $i$ is in the working state. Thus, node $i$ will never execute a rule again. If node $i$ is attacked, it will be locked by $r_{2}$ and remains in its state until its healing/recovery. After that, it will join the set of sleeping nodes.

Lemma 2 If a sensor node is enabled by rule $r_{2}$, then each one of its neighbors will execute at most one more rule until their next wakeup/probing, and this rule will be $r_{1}$.

PROOF Let $i$ be a node that executes $r_{2}$. When node $i$ changes to working state, all its neighbors are either in sleeping or probing or locked state. So we have 
three possible scenarios: i) neighbors in sleeping state: there is no conflict in this case. ii) neighbors with probing state: those neighbors have a higher $I d$ than $i$. iii) locked neighbors will remain in their state until their healing/recovery before joining the set of sleeping nodes.

Lemma 3 Every sensor node is either independent or dominated or locked.

Proof From the point of view of node $i$, we have three scenarios:

- if node $i$ is in the working state and is not independent, then $i$ may execute rule $r_{3}$.

- if node $i$ is in the sleeping $\vee$ probing state and is not dominated, then node $i$ may execute rule $r_{2}$.

- if node $i$ is in the locked state, then node $i$ will remain in its state until its healing/recovery.

Lemma 4 When a node is not locked $\vee$ sleeping, it can make at most 2 moves.

Proof By Lemma 1 and Lemma 2, each rule can be executed at most once by a node. Hence, the only case a node makes two moves is when it executes $r_{3}$ then $r_{2}$ with a working state.

Theorem 1 With respect to the legitimate state $\mathcal{L}$ of the network, the proposed algorithm converges within $2 n$ moves.

Proof This follows from Lemma 1 to Lemma 4.

\section{Simulations}

This section is dedicated to the evaluation of the SIR approach through experiments. We will show, using both the mathematical modeling and a basic wireless sensor network designed in Python, that taking place in the conditions of Proposition 4 is a guarantee to achieve information survivability in WSNs.

\subsection{Mathematics-based simulations}

In this first illustration written in Python language, the initial number of susceptible sensors is set to 300 while 3 nodes initially receive the datum. System 8 is then discretized and 4 experiments have been conducted, leading twice to the situation $R_{0}<1$, and twice to the opposite situation.

Figure 12 shows the obtained result. We can see that the $I$ compartment is never empty when $R_{0}>1$, leading to a data survivability in this SIR model simulation. Conversely, when $R_{0}<1$, the information is obviously lost. 


\subsection{Networks simulation using Python}

In this second set of experiments, we show that the time period of the presence of the information can be extended in a wireless sensor network simulated with Python, and when satisfying Proposition 4

We have firstly deployed $N=100$ sensors, all belonging in the susceptible compartment, and with respect to the algorithm detailed in the previous section. In the initial condition, each sensor has a probability of $10 \%$ to have detected an intrusion (this is the information). At each time unit, an average of $l N$ new sensors are awaken. For each informed sensor and for each of its susceptible neighbor, the data is sent with a probability $b I$. The death rate of each sensor is set to $m$ (each awaken sensor has the probability $m$ to empty its battery during the considered time unit), while each informed sensor has a probability $c$ to loose the information (to move in the $\mathrm{R}$ compartment). The whole network is observed during 60 time units.

We have firstly set $l=0.017, m=0.0018, c=0.035$, and $b=0.33$, which leads to $R_{0}=84.69$, and to the situation depicted in Figure 13(a) In this experiment, ${ }^{*}$ symbols have been used for the susceptible sensors, $\times$ for the informed ones, a circle is for the recovered ones, while the straight line counts the number of dead sensors. A second set of parameters has led to $R_{0}=0.06$, and to the situation described in Figure $13(\mathrm{~b})$.

\subsection{0 experiments with random parameters}

We have then launched the previous simulator 100 times with random parameters. At each simulation, probability $l$ is randomly picked in the interval [0,0.2[, $m$ is chosen in [0,0.01], $c$ is picked in [0,0.1], while $b$ is in [0,0.033[, in order to be close to a real situation while having $R_{0}<1$ and $R_{0}>1$ both represented. During these 100 experiments, we have obtained 39 times the situation $R_{0}<1$ with an average of 0.34 (and 61 times the situation $R_{0}>1,16.05$ of average).

We found an average number of informed sensors equal to 15.50 in the first situation, while it is the double in the second one (33.12 informed sensors in average). In 7 of the 39 simulations with $R_{0}<1$ (17.95\%), the number of informed sensors has reached 0 , while the information has disappeared 2 times during the 61 other simulations $(3.27 \%)$. The minimum of informed sensors is attained at the 35-th time unit (in average) in the first situation, while we reach it earlier in the second one (31-th time unit).

To sum up, the information has disappeared in $3.27 \%$ of the simulations when $R_{0}>1$, while it has been lost in $17.95 \%$ of the cases in the second situation.

\section{Conclusion}

This paper presented an efficient technique that uses epidemic domain models in the context of data survival in unattended WSNs. We studied two models (SIR and SIS) that can ensure the survivability of the datum in presence of different types of attacks. We showed that our method is well adapted to UWSN 
scenarios. In a second step, we proposed and analyzed an efficient distributed algorithm to tackle the problem of data survivability. In future work, the authors' intention is to take into account the possibility of aggregation layers in the wireless sensor networks [1]: aggregators could transfer only one alert signal for all their neighborhood.

\section{References}

[1] Jacques Bahi, Christophe Guyeux, and Abdallah Makhoul. Two security layers for hierarchical data aggregation in sensor networks. IJAACS, International Journal of Autonomous and Adaptive Communications Systems, $*(*): * * * \_* * *, 2011$. Accepted manuscript. To appear.

[2] Jacques Bahi, Christophe Guyeux, Abdallah Makhoul, and Congduc Pham. Low cost monitoring and intruders detection using wireless video sensor networks. International Journal of Distributed Sensor Networks, 2012, 2012. 11 pages.

[3] Jacques Bahi, Abdallah Makhoul, and Maguy Medlej. Data aggregation for periodic sensor networks using sets similarity functions. IWCMC 2011, 7th IEEE Int. Wireless Communications and Mobile Computing Conference, pages 559-564, 2011.

[4] Jacques Bahi, Abdallah Makhoul, and Maguy Medlej. An optimized innetwork aggregation scheme for data collection in periodic sensor networks. ADHOC-NOW 2012, 11-th Int. Conf. on Ad Hoc Networks and Wireless, pages 153-166, 2012.

[5] Jacques M. Bahi, Abdallah Makhoul, and Ahmed Mostefaoui. Hilbert mobile beacon for localisation and coverage in sensor networks. Int. J. Systems Science, 39(11):1081-1094, 2008.

[6] Jacques M. Bahi, Abdallah Makhoul, and Ahmed Mostefaoui. Localization and coverage for high density sensor networks. Computer Communications, 31(4):770-781, 2008.

[7] Deepayan Chakrabarti, Jure Leskovec, Christos Faloutsos, Samuel Madden, Carlos Guestrin, and Michalis Faloutsos. Information survival threshold in sensor and p2p networks. Proceedings of 26th Annual IEEE ICC, 2007.

[8] Roberto Di Pietro, Luigi V. Mancini, Claudio Soriente, Angelo Spognardi, and Gene Tsudik. Catch me (if you can): Data survival in unattended sensor networks. pages 185-194, 2008.

[9] Roberto Di Pietro, Luigi V. Mancini, Claudio Soriente, Angelo Spognardi, and Gene Tsudik. Playing hide-and-seek with a focused mobile adversary in unattended wireless sensor networks. Ad Hoc Netw., 7(8):1463-1475, 2009 . 
[10] Roberto Di Pietro and Nino Vincenzo Verde. Epidemic data survivability in unattended wireless sensor networks. pages 11-22, 2011.

[11] R. Guha, R. Kumar, P. Raghavan, and A. Tomkins. Propagation of trust and distrust. $W W W, 2004$.

[12] Herbert W. Hethcote. The mathematics of infectious diseases. SIAM Review, 42:599-653, 2000.

[13] W. O. Kermack and Ag McKendrick. A Contribution to the Mathematical Theory of Epidemics. Proceedings of the Royal Society of London. Series A, Containing Papers of a Mathematical and Physical Character, 115(772):700-721, August 1927.

[14] Charalampos Konstantopoulos, Grammati E. Pantziou, Damianos Gavalas, Aristides Mpitziopoulos, and Basilis Mamalis. A rendezvous-based approach enabling energy-efficient sensory data collection with mobile sinks. IEEE Trans. Parallel Distrib. Syst., 23(5):809-817, 2012.

[15] Arun K. Kumar and Krishna M. Sivalingam. Energy-efficient mobile data collection in wireless sensor networks with delay reduction using wireless communication. COMSNETS, pages 1-10, 2010.

[16] Di Ma and Gene Tsudik. Dish: Distributed self-healing. pages 47-62, 2008.

[17] R. Di Pietro, L. V. Mancini, C. Soriente, A. Spognardi, and G. Tsudik. Data security in unattended wireless sensor networks. IEEE Transactions on Computers, 58:1500-1511, 2009.

[18] Roberto Di Pietro and Nino Vincenzo Verde. Epidemic theory and data survivability in unattended wireless sensor networks: Models and gaps. Pervasive and Mobile Computing, 9(4):588 - 597, 2013.

[19] W. REN, J. ZHAO, and Y. REN. Network coding based dependable and efficient data survival in unattended wireless sensor networks. Journal of Communications, 4, 2009.

[20] Mateus A. S. Santos and Cintia Borges Margi. Design and implementation of data survival in unattended wireless sensor networks. IEEE International Performance Computing and Communications Conference, 0:1-6, 2011.

[21] Ryo Sugihara and Rajesh K. Gupta. Improving the data delivery latency in sensor networks with controlled mobility. DCOSS, pages 386-399, 2008.

[22] Ryo Sugihara and Rajesh K. Gupta. Optimal speed control of mobile node for data collection in sensor networks. IEEE Trans. Mob. Comput., $9(1): 127-139,2010$. 
[23] Kaiping Xue, Changsha Ma, Peilin Hong, and Rong Ding. A temporalcredential-based mutual authentication and key agreement scheme for wireless sensor networks. Journal of Network and Computer Applications, 36(1):316-323, 2013.

[24] Yanli Yua, Keqiu Lia, Wanlei Zhoub, and Ping Li. Trust mechanisms in wireless sensor networks: Attack analysis and countermeasures. Journal of Network and Computer Applications, 35(3):867-880, 2012.

[25] YoungSang Yun, Ye Xia, Behnam Behdani, and J. Cole Smith. Distributed algorithm for lifetime maximization in a delay-tolerant wireless sensor network with a mobile sink. IEEE Trans. Mob. Comput., 12(10):1920-1930, 2013.

[26] D. Zeinalipour-Yazti, V. Kalogeraki, D. Gunopulos, A. Mitra, A. Banerjee, and W. Najjar. Towards in-situ data storage in sensor databases. in Proc. of 10th Panhellenic Conference on Informatics (PCI'05), pages 36-46, 2005. 


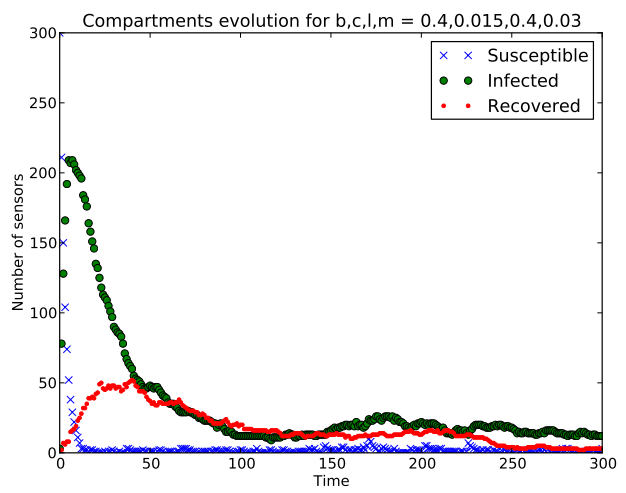

(a) $R_{0}=118.51$

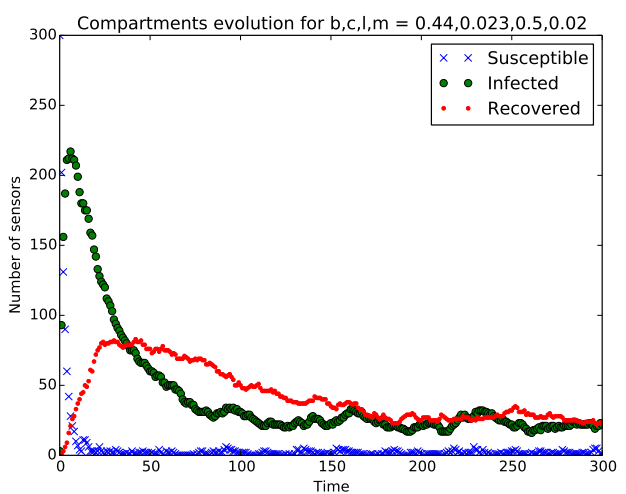

(b) $R_{0}=255.81$

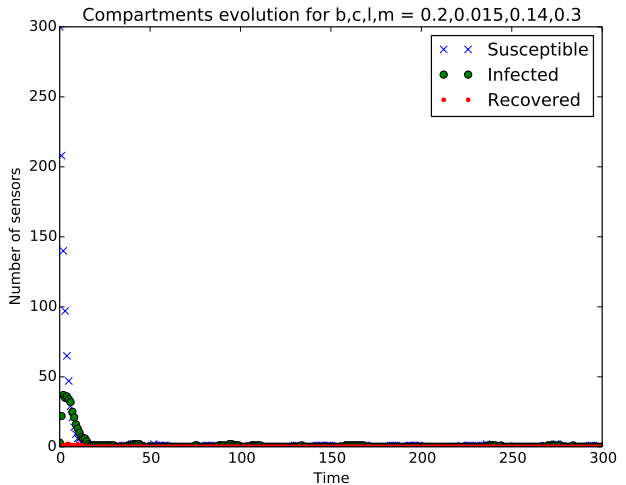

(c) $R_{0}=0.29$

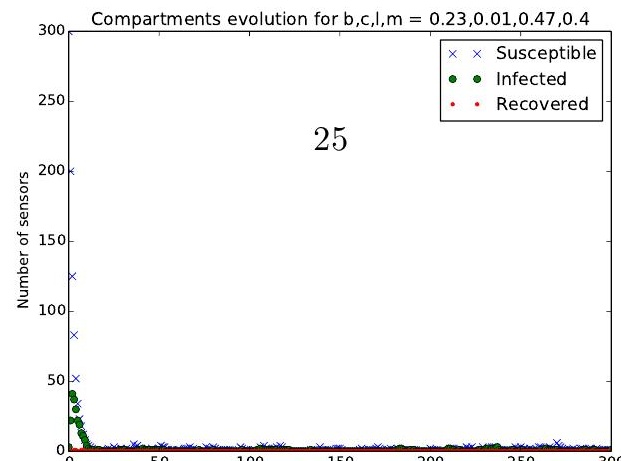




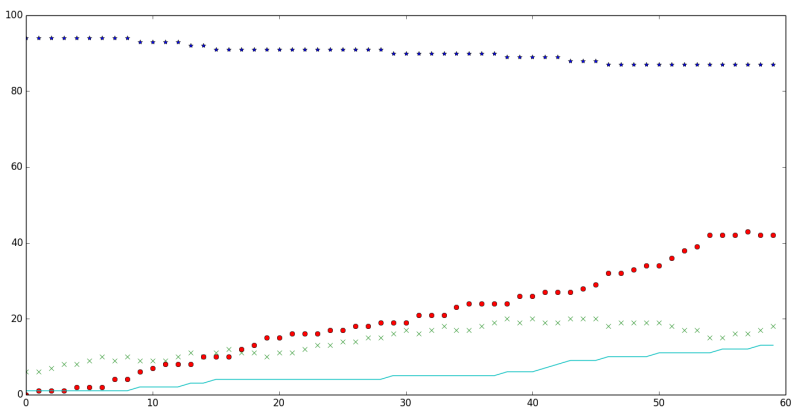

(a) $R_{0}=84.69$

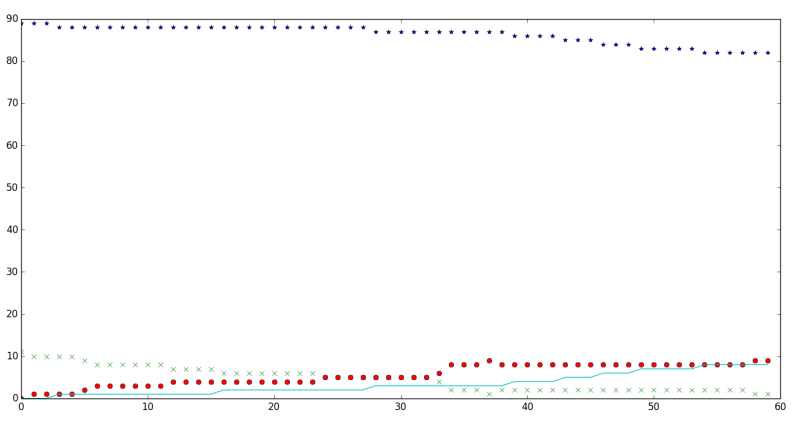

(b) $R_{0}=0.06$

Figure 13: Simulation of a wireless sensor network 